Results Forty-five recommendations spanning nine CPGs were processed and converted into Drools rules. We identified 138 decision variables and 91 actions within the selected recommendations. From these, we encoded 148 concepts associated with value set meta-tags and 238 decision rules.

Discussion The level of difficulty required to encode the recommendations was directly related to the specificity, complexity, and decidability of each recommendation; there was significant variability among the recommendations.

Implications for Guideline Developers/Users CPG developers may need new processes in order to optimise recommendations for incorporation into CDS systems.

\section{P151 CULTURE AND GUIDELINES: HOW CULTURAL DIFFERENCES IN TREATMENT APPROACH AFFECT INTERPRETATION OF LITERATURE AND GUIDELINE RECOMMENDATIONS}

'S Schwartz, ${ }^{2} \mathrm{C}$ Drumheller. "Virginia Mason Medical Center, Seattle, USA; ${ }^{2}$ American Academy of Otolaryngology - Head and Neck Surgery, Alexandria, USA

\section{0:1136/bmjqs-2013-002293.182}

Background Guideline development activity is primarily focused in Western Europe and North America. Consequently, western medical interventions and approaches are disproportionately represented among guideline developers. scepticism about cultural variations in treatment, concern of publication bias in specific regions or languages, and resulting scepticism of foreign literature compounds the problem of accurately assessing evidence and making sound recommendations. When accounting for publication bias and/or prevailing cultural paradigms, guideline developers may struggle to determine the benefit-harm ratio of alternative/complementary interventions.

Context A recent guideline development panel struggled with precisely these issues when reviewing available literature to formulate a recommendation on acupuncture therapy for Bell's palsy patients. All physicians on the panel practiced medicine in the United States, and were unfamiliar with acupuncture therapy. Available literature came predominantly from one country with evidence of severe publication bias. The panel was unable to determine the benefit-harm ratio of acupuncture therapy, and ultimately could make no recommendation for the use of acupuncture for Bell's palsy patients.

Description of Best Practice Guideline developers need to give careful consideration to interpretation of literature when there may be significant cultural differences in treatment approach, cultural bias among the panel, or publication bias that may affect recommendations. Transparent discussion that recognises these issues will help ensure that recommendations regarding alternative/complementary interventions are sound.

Lesson for Guideline Developers Guideline developers need to be aware of potential bias as to how cultural differences in treatments are represented in guideline recommendations, and be mindful regarding the cross-cultural applicability of guideline content.

\section{P153 SUCCESSFUL STRATEGIES IN THE IMPLEMENTATION OF CLINICAL PRACTICE GUIDELINES: CREATING EVIDENCE- INFORMED PRACTICE CULTURES}

H McConnell, D Grinspun, I Bajnok. Registered Nurses' Association of Ontario, Toronto, Canada

10:1136/bmjqs-2013-002293.183
For over a decade, a professional nursing association has lead a programme focused on the development, dissemination, implementation and evaluation of clinical and healthy work environment guidelines. This programme has achieved considerable recognition around the globe based on its rigorous guideline development process, and innovative implementation strategies. These strategies are founded on an evidence-informed implementation model, and include individual, organisational and system level approaches. A key organisational level strategy within this programme, the Best Practice Spotlight Organization (BPSO) initiative, was designed to support health care organisations in their journey towards clinical excellence through the implementation and sustainability of multiple clinical practice guidelines. This initiative was launched in 2003 with nine organisations (acute care hospitals and home health care agencies), and has grown to include 68 BPSOs representing 294 sites. This reach has included international BPSOs in Spain, Australia, Chile, and the United States. The BPSO initiative provides specific coaching, mentoring, knowledge transfer and capacity building opportunities, and support to leaders in the BPSO sites as they implement, evaluate and work to sustain clinical guidelines both across their organisations and at the team/unit level. This strategic approach has served to trigger the development of evidence informed cultures, improve patient care and enrich the professional practice of nurses and other health care providers. This presentation will share some of the key outcomes of this guideline implementation strategy, and will highlight success storeys of how BPSOs are changing the nursing and health care landscape to foster a culture of evidence informed practice.

\section{P154 DEVELOPMENT OF THE DOCUMENTATION AND APPRAISAL REVIEW TOOL (DART) FOR SYSTEMATIC REVIEWS}

${ }^{1} \mathrm{R}$ Diekemper, ${ }^{2} \mathrm{~B}$ Ireland, ${ }^{3} \mathrm{~L}$ Merz. ${ }^{1}$ American College of Chest Physicians, Northbrook, USA: ${ }^{2}$ The Evidence Doc, Pacific, USA; ${ }^{3} B J C$ HealthCare Center for Clinical Excellence, St. Louis, USA

\section{0:1136/bmjqs-2013-002293.184}

Background Systematic reviews are the foundation for evidencebased guidelines. Rigorous standards exist, but there is wide variation in implementation, highlighting the need for a more comprehensive quality assessment tool for systematic reviews.

Objective To develop a tool that sufficiently evaluates major biases relevant to experimental and observational study designs. Methods The Documentation and Appraisal Review Tool (DART) was developed using epidemiologic principles of study design and the following resources: Overview Quality Assessment Questionnaire (OQAQ), Assessment of Multiple Systematic Reviews (AMSTAR), the Cochrane Handbook, and the standards promoted by the Agency for Healthcare Research and Quality, and the Institutes of Medicine (IOM). DART underwent multiple rounds of testing and revisions.

Results Compared to OQAQ and AMSTAR, DART includes two unique questions and several questions covered by OQAQ or AMSTAR but not both. OQAQ and DART had the highest reporting consistency. Four AMSTAR questions elicited inconsistent responses. Identifying reviewer rationale was most difficult using the OQAQ tool, and easiest using DART.

Discussion DART allows for documentation of reviewer rationale, facilitating reconciliation between reviewers and documentation for future updates. DART also allows for evaluation of major biases relevant to observational study designs and the 